OPEN ACCESS

Edited by:

William Small, Jr.,

Loyola University Chicago,

United States

Reviewed by:

Tsair-Fwu Lee,

National Kaohsiung University of Science and Technology, Taiwan

Michael Eble,

University Hospital RWTH Aachen,

Germany

*Correspondence:

Jian-Xiong Wu

dr.wujx@hotmail.com

Qinfu Feng

qinfufeng19@163.com

${ }^{+}$These authors have contributed equally to this work

Specialty section:

This article was submitted to

Radiation Oncology,

a section of the journal

Frontiers in Oncology

Received: 09 September 2021 Accepted: 07 January 2022

Published: 11 February 2022

Citation:

Wu Y-L, Zhai YR, Li MH, Cai J-Q, Ma $P$, Wang $L-M$, WU $X-H$, Wang $X-D$, Wu F, Zeng Q, Chen B, Li Y -X, Wu J-X and Feng QF (2022) Long-Term

Outcome of Centrally Located Hepatocellular Carcinomas

Treated by Radical Resection Combined With Intraoperative Electron Radiotherapy (IOERT).

Front. Oncol. 12:773301. doi: 10.3389/fonc.2022.773301

\section{Long-Term Outcome of Centrally Located Hepatocellular Carcinomas Treated by Radical Resection Combined With Intraoperative Electron Radiotherapy (IOERT)}

\author{
Yan-Ling Wu ${ }^{1 \dagger}$, Yirui Zhai ${ }^{1 \dagger}$, Minghui $\mathrm{Li}^{1}$, Jian-Qiang Cai ${ }^{2}$, Pan Ma ${ }^{1}$, Li-Ming Wang ${ }^{2}$, \\ Xiu-Hong $W u^{3}$, Xiao-dan Wang ${ }^{1}$, Fan $W^{2}{ }^{2}$, Qiang Zeng ${ }^{1}$, Bo Chen ${ }^{1}$, Ye-Xiong $L^{1}{ }^{1}$, \\ Jian-Xiong $W_{u^{2 *}}$ and Qinfu Feng ${ }^{1 *}$

\begin{abstract}
${ }^{1}$ Department of Radiation Oncology, National Cancer Center/National Clinical Research Center for Cancer/Cancer Hospital, Chinese Academy of Medical Sciences (CAMS) and Peking Union Medical College (PUMC), Beijing, China, ${ }^{2}$ Department of Hepatobiliary Surgery, National Cancer Center/National Clinical Research Center for Cancer/Cancer Hospital, Chinese Academy of Medical Sciences (CAMS) and Peking Union Medical College (PUMC), Beijing, China, ${ }^{3}$ Department of Operating Room, National Cancer Center/National Clinical Research Center for Cancer/Cancer Hospital, Chinese Academy of Medical Sciences (CAMS) and Peking Union Medical College (PUMC), Beijing, China
\end{abstract}

Purpose: To explore the feasibility and safety of centrally located hepatocellular carcinoma (CL-HCC) treated by narrow-margin resection combined with intraoperative electron radiotherapy (IOERT).

Methods and Materials: From November 2009 to November 2016, 37 consecutive patients were treated with IOERT as adjuvant treatment during narrow-margin resection for CL-HCC. Long-term outcomes, adverse events for surgery, and acute and chronic toxicities were analyzed.

Results: The median follow-up was 57.82 months (range, 3.75-111.41 months). A total dose of 15 Gy (range 12 to $17 \mathrm{~Gy}$ ) (prescribed at the $90 \%$ isodose) was delivered with a $0.9 \mathrm{~cm}$ (range 0.8-1.2 cm) median treatment depth targeting the narrow-margin. The 1year, 3-year and 5-year OS rates were 91.39\%, 88.34\% and 88.34\%, respectively. The 1year, 3-year and 5-year DFS rates were $80.81 \%, 68.59 \%$ and $54.17 \%$, respectively. In the univariate analysis, none of the treatment characteristics were predictive of overall survival. Fifteen (40.5\%) patients suffered from a recurrence event. No patient had marginal recurrence. The 1-year, 3-year and 5-year intrahepatic recurrence rates were 19.75\%, $25.92 \%$ and $39.58 \%$, respectively. The 1-year, 3-year and 5-year extrahepatic recurrence rates were $2.7 \%, 5.95 \%$ and $9.87 \%$, respectively. There was no 30-day surgical-related death. Three patients had grade 4 , and 28 patients had grade 3 alanine aminotransferase (ALT) levels, and seven patients had grade 4, and 30 patients had grade 3 aspartate transaminase (AST) levels. All of them returned to normal within four months. There was no acute radiation-induced liver injury during follow-up. There were no acute or chronic toxicities associated with IOERT. 


\begin{abstract}
Conclusion: IOERT for narrow-margin CL-HCC may achieve good long-term survival outcomes, without significantly increasing acute and chronic toxicities. An IOERT dose of 15Gy may be the safest and most feasible. IOERT might be considered as an adjuvant therapy for CL-HCC patients with a narrow-margin.
\end{abstract}

Keywords: centrally located hepatocellular carcinoma (CL-HCC), intraoperative electron radiotherapy (IOERT), safety, prognosis, narrow-margin resection

\section{INTRODUCTION}

Hepatocellular carcinoma (HCC) is the third cause of cancerrelated deaths worldwide. The incidence and mortality of HCC in China ranks the first in the world, and accounts for approximately half of all new HCC cases (1). Surgical resection is the main treatment method for HCC patients when liver transplantation is unavailable (2). However, long-term outcomes remain poor due to a high recurrence rate (5-year recurrence rate of nearly 70\%) (3).

Centrally located hepatocellular carcinoma (CL-HCC) is a tumor located at the bifurcation of the portal vein, the confluence of the three main hepatic veins, the inferior vena cava or within 1 centimeter $(\mathrm{cm})$ of the posterior inferior vena cava trunk. Due to its proximity to important blood vessels and bile ducts, ensuring a safe surgical resection margin of $1 \mathrm{~cm}$ is difficult. Narrower resection margins $(<1 \mathrm{~cm})$ and even null margins after surgery greatly increase the possibility of recurrence after surgery. It is reported that the recurrence rate of central liver cancer after five years is more than $90 \%$, and the five-year DFS is $15-30 \%(4,5)$. It is therefore paramount, to find efficacious adjuvant therapies that reduce CL-HCC recurrence.

HCC is sensitive to radiation with a sensitivity equivalent to squamous cancer cells. The local control rate of HCC is $71-100 \%$ after radical radiotherapy (6-9). Considering that the tumor bed tissue is at the highest risk of residual subclinical tumor cells, a local dose escalation has been shown to decrease CL-HCC recurrence rates most effectively (7). Intraoperative electron radiotherapy (IOERT) delivered immediately after surgery can reduce the spread of cancer cells. Direct visualization during surgery guarantees an accurate dose delivery to the tumor bed and provides greater protection of the normal tissue. In contrast to external-beam radiation therapy (EBRT), a single high-dose of radiation produces a higher biological effect and has the advantages of eliminating the residual micro-lesions in the tumor bed, and at the same time, intra- and interfractional motion is reduced and the dose applied more homogeneously. This technique provides more patient comfort and reduces total treatment time by one to five weeks (10-18).

IOERT as an adjuvant therapy has been used to treat head and neck, breast, prostate and gastrointestinal cancers, as well as retroperitoneal and extremity sarcomas (10-18). However, the application of IOERT is not well understood in CL-HCC patients. It is reasonable to hypothesize that IOERT may improve local control, and therefore, survival in CL-HCC patients. This study assesses the long-term survival and safety among CL-HCC patients who received adjuvant IOERT.

\section{MATERIALS AND METHODS}

\section{Patient Selection}

This pilot study was approved by the ethics committee of the Cancer Hospital of the Chinese Academy of Medical Sciences (NCC2013RE-079). The inclusion criteria were: (1) two kinds of imaging examinations or pathologically confirmed CL-HCC prior to surgery; (2) no preoperative radiofrequency, ablation, intervention or chemoradiotherapy; (3) single or multiple lesions limited to three liver segments and able to be completely resected; (4) a resection margin of $<1 \mathrm{~cm}$; (5) no residual tumor with intraoperative frozen pathology, and enough liver tissue to ensure function; (6) an Eastern Cooperative Oncology Group (ECOG) score $\leq 1$, a Child-Pugh score of A, ICG clearance (ICG. R 15) < 15\%; and (7) no heart, lung, kidney, or other serious surgical complications.

From October 2009 to November 2016, the clinical records of 37 consecutive patients diagnosed with CL-HCC treated with IOERT as adjuvant treatment during narrow-margin resection were retrospectively reviewed in this prospectively assembled database. The database tracks patient medical history, family history, comorbidities, clinical history, tumor characteristics, diagnostic tests, therapeutic interventions, complications, and outcomes.

\section{Treatment}

Pretreatment evaluation included medical history and physical examination, serum biochemistry for liver function status, clear imaging of the tumor, blood vessels and bile duct with pelvis multiphase contrast-enhanced computed tomography (CT), magnetic resonance imaging (MRI) or ultrasound. Surgery and radiotherapy experts identified those suitable for IOERT. Patients were informed of the possible benefits and potential risks. All patients provided written informed consent.

\section{Surgery}

Preoperative examination and an exploratory laparotomy were conducted to assess whether the tumor had metastasized, the degree of hepatic cirrhosis, tumor location, tumor size, the relationship between the tumor, important blood vessels and the bile ducts. Ultrasound examination was used to diagnose portal vein or major artery invasion. The extent of resection included the tumor and at least $1 \mathrm{~cm}$ of the surrounding tissue to achieve mesohepatectomy, two- or three-segment resection, segmentectomy or non-anatomical hepatectomy according to the National Comprehensive Cancer Network guidelines (19). 
Intraoperative ultrasonography and a cavitron ultrasonic surgical aspirator were used to carefully dissect and peel away the tumor from the major vascular surface. If resection of the major vessels was unavoidable, revascularization was performed to preserve more liver tissue and prevent postoperative liver failure. After the tumor was completely resected, the frozen resection margin was sent for a quick pathological examination to confirm there was no residual tumor before starting IOERT. Following IOERT, the abdomen was closed after the confirmation of no active liver bleeding, biliary fistulas and normal color of the remaining liver.

\section{Intraoperative Electron Radiotherapy}

IOERT was initiated after confirmation of the target volume by radiotherapists and surgeons. The resected margin receiving IOERT, only extended to the high risk area near to the major vasculature, duct structures and the deep resected margin. The surface resection margins were at a sufficient safety distance. A smaller target volume reduced the possibility of radiationinduced liver damage. The desired IOERT field size and depth were achieved via the most appropriate angle and diameter of the applicator tube, electron beam energy, and the covering of bolus while minimizing the exposure of the adjacent normal tissue.

The applicator tube was placed under visual control and ranged from 3 to $10 \mathrm{~cm}$ in diameter, and was available in $0.5 \mathrm{~cm}$ steps with $0^{\circ}, 15^{\circ}$, and $30^{\circ}$ angles. High energy electron $(6 / 9 \mathrm{MeV})$ beam radiotherapy was delivered with a total dose of $15 \mathrm{~Gy}$ (range from 12 to $17 \mathrm{~Gy}$ ) (prescribed at the $90 \%$ isodose) with a $0.9 \mathrm{~cm}$ (range, $0.8-1.2 \mathrm{~cm}$ ) median treatment depth targeting the narrow-margin. IOERT was administered using a Mobetron 1000 mobile intraoperative radiotherapy accelerator (Intraop Medical Corporation, Sunnyvale, CA) in the operating room (See Figure 1).

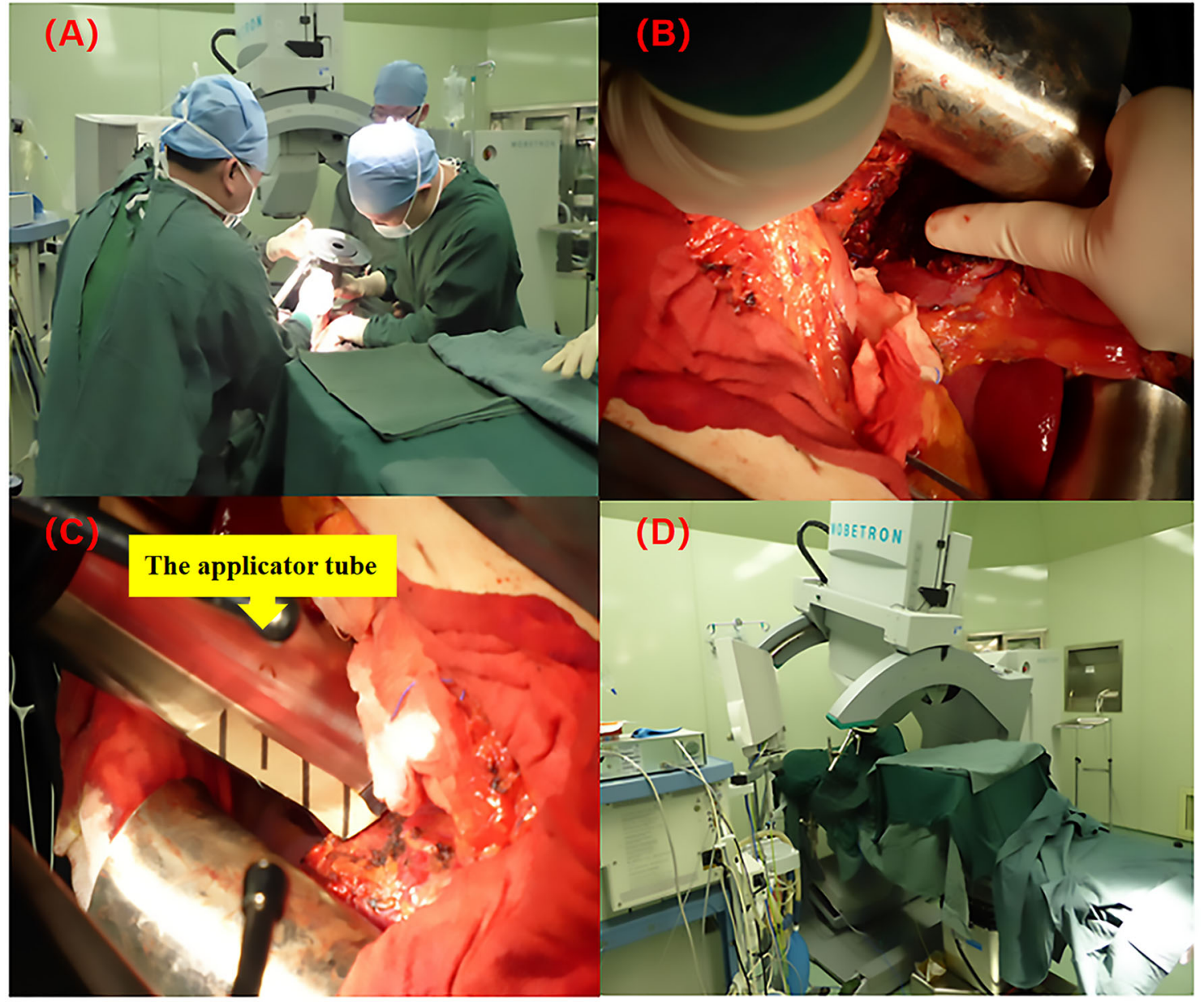

FIGURE 1 | The IOERT process using the Mobetron 2000 mobile electron accelerator. (A) Radiotherapists and surgeons are confirming the target volume of IOERT; (B) The resected margin receiving IOERT, only extended to the high risk area near to the major vasculature, duct structures and the deep resected margin (the direction of the finger); (C) The applicator tube was placed under visual control (the diameter of applicator tube was ranged from 3 to $10 \mathrm{~cm}$, and was available in $0.5 \mathrm{~cm}$ steps with $0^{\circ}, 15^{\circ}$, and $30^{\circ}$ angles); (D) IOERT was administered using a Mobetron 1000 mobile intraoperative radiotherapy accelerator (Intraop Medical Corporation, Sunnyvale, CA). 


\section{Toxicity Criteria}

Acute toxicity was evaluated one month after IOERT. Chronic toxicity was defined as a morbidity occurring at least one month after IOERT. Acute and chronic radiation toxicities were evaluated according to the Radiation Therapy Oncology Group (RTOG) and the European Organization for Research and Treatment of Cancer (EORTC) common Toxicity Criteria, version 4.0 (CTCAE 4.0) (Cancer Therapy Evaluation Program, Common Terminology Criteria for Adverse Events, Bethesda, MD, USA).

Radiation-induced liver disease (RILD) was defined as having an elevated anicteric alkaline phosphate level at least two times of the pre-treatment level and non-malignant ascites (classic RILD) (20), or, elevated transaminases at least five times the pretreatment level (non-classic RILD) (21) in the absence of documented progressive disease. Patients were evaluated for RILD four months after radiotherapy. RILD was excluded from liver injury caused by drug hepatitis, interventional therapy, an acute outbreak of viral hepatitis, obstructive jaundice, and intrahepatic tumor progression.

\section{Recurrence Criteria}

Recurrence was evaluated by clinical follow-up and imaging. Abdominal ultrasound, enhanced CT and MRI were used to find new intrahepatic, extrahepatic nodules or features of tumor malignancy. If there was no tumor recurrence by imaging examination but the alpha-fetoprotein (AFP) was larger than $400 \mathrm{ug} / \mathrm{L}$ for more than four weeks or $200 \mathrm{ug} / \mathrm{L}$ for more than eight weeks after excluding for a reproductive system tumor, pregnancy, and hepatitis, a diagnosis of tumor recurrence was made. Recurrence was divided into intrahepatic and extrahepatic relapse, marginal recurrence, and early and late recurrence. The criteria for marginal recurrence were defined on whether recurrence was inside the $1 \mathrm{~cm}$ surgical margin area, since $0.9 \mathrm{~cm}$ was the median IOERT depth. Early recurrence was defined as within 18 months. Late recurrence was defined as 18 months or over (22).

\section{Follow-Up and Statistical Analysis}

Follow-up was every three months during the first two years, and every six months thereafter. Follow-up included: routine blood work, biochemical, blood clotting and tumor markers and abdominal CT/MRI/B ultrasound analysis. Overall survival (OS) was calculated as the time from surgical resection to death of any cause. DFS was calculated as the time from surgical resection to intrahepatic or extrahepatic recurrence of CL-HCC.

Statistical analyses were performed using SPSS v22.0 (IBM Corp, Armonk, NY). The counted data were analyzed by the T test, and the $\mathrm{X}^{2}$ test. Survival rates were calculated using the Kaplan-Meier method and intergroup differences compared using the log-rank test. Prognostic factors for OS were evaluated at the univariate level using the Cox proportional hazards models. A two-sided $\mathrm{P}$ value $<0.05$ was considered statistically significant in all the analyses.

\section{RESULTS}

\section{Patients, Tumors and Treatment Characteristics}

The median follow-up was 57.82 months (range, 3.75-111.41 months). Thirty-two patients $(86.5 \%)$ were male. The median age was 58 years old (range, 37-75 years old). Risk factors included long-term drinking history $(\mathrm{n}=5)$, HBV positive status $(n=27)$ and cirrhosis $(n=34)$. All had Child-Pugh grade A liver function. A tumor marker AFP value $\geq 400 \mathrm{ug} / \mathrm{L}$ was present in 11 patients. Thirty-three patients had single lesions, and four patients had multiple intrahepatic lesions. According to the American Joint Commission on Cancer (AJCC, $7^{\text {th }}$ ed.) staging system for HCC, stage T1, T2 and T3 were recorded as $20(54.1 \%), 13(35.1 \%)$ and $4(10.8 \%)$, respectively. Nine patients had tumor infiltration/compression of the major vasculature, nine patients had microvascular invasion of the tumor, and 22 patients had liver capsule invasion of the tumor.

The median operation time was 285 minutes (range, 195-510 minutes). One patient had pleural effusion after surgery which improved after thoracic puncture and drainage. One patient developed acute renal failure on the second day and received renal replacement therapy and returned to normal within one week. There was no pulmonary infection, abdominal infection, incision infection, massive bleeding, or death within 30 days of surgery. The median tumor diameter was $5 \mathrm{~cm}$ (range, $2-15 \mathrm{~cm}$ ). Fourteen patients had a diameter $>5 \mathrm{~cm}$ and 23 patients $\leq 5 \mathrm{~cm}$. The median applicator diameter was $5.5 \mathrm{~cm}$ (range, $3-10 \mathrm{~cm}$ ). The median IOERT dose was 15Gy (range, 12-17Gy), three patients received $\leq 15 \mathrm{~Gy}, 23$ patients received $15 \mathrm{~Gy}$, and 11 patients $>15 \mathrm{~Gy}$. For patients' baseline, tumors and treatment characteristics, see Table 1.

\section{Survival Rates}

By the end of the follow-up time, five patients (13.5\%) were dead, four of which $(10.8 \%)$ died from HCC related failure. Among them, three died from liver failure caused by intrahepatic recurrence and subsequent tumor-related treatment. One patient died from systemic multi-organ failure caused by multiple metastasis, and one patient with multiple bone metastasis who was stable after treatment died from respiratory failure due to poor lung function. The 1-year, 3year and 5-year OS were 91.39\%, 88.34\% and 88.34\%, respectively (see Figure 2A). The 1-year, 3-year and 5-year DFS were $80.81 \%, 68.59 \%$ and $54.17 \%$, respectively (see Figure 2B).

\section{Univariate Analysis}

We analyzed which patient characteristics and treatment parameters might be associated with survival outcomes (see Table 2). Among patient characteristics, the following factors entered the univariate survival analysis and demonstrated no statistical significance: age $(P=0.291)$, gender $(P=0.319)$, hepatitis $\mathrm{B}$ virus $(\mathrm{HBV})(\mathrm{P}=0.372)$, long-term drinking $(\mathrm{P}=$ $0.693)$, AFP value $(P=0.237)$, tumor size $(P=0.17)$, histological 
TABLE 1 | Demographic and clinicopathological features of the 37 patients with radical resection combined with IOERT.

\begin{tabular}{|c|c|c|}
\hline Variables & Level & No. of patients (\%) \\
\hline \multirow[t]{3}{*}{ Age (years) } & Median (min-max) & $58(37 \sim 75)$ \\
\hline & $\leq 60$ & $23(62.2)$ \\
\hline & $>60$ & $14(37.8)$ \\
\hline \multirow[t]{2}{*}{ Gender } & Male & $32(86.5)$ \\
\hline & Female & $5(13.5)$ \\
\hline \multirow[t]{2}{*}{ Chronic hepatitis } & $\mathrm{HBV}+$ & $27(73)$ \\
\hline & Cirrhosis & 34 (91.9) \\
\hline \multirow[t]{2}{*}{ Long-term drinker } & yes & $5(13.5)$ \\
\hline & no & $32(86.5)$ \\
\hline \multirow[t]{2}{*}{ AFP level (ug/L) } & $<400$ ug/L & $26(70.3)$ \\
\hline & $\geq 400 \mathrm{ug} / \mathrm{L}$ & $11(29.7)$ \\
\hline \multirow[t]{2}{*}{ Tumor diameter size (cm) } & $\leq 5 \mathrm{~cm}$ & $23(62.2)$ \\
\hline & $>5 \mathrm{~cm}$ & $14(37.8)$ \\
\hline \multirow[t]{2}{*}{ Number of primary tumors } & Single & $33(89.2)$ \\
\hline & Multiple & $4(10.8)$ \\
\hline \multirow[t]{2}{*}{ Histopathology grade (WHO) } & Well-Mid & $26(70.3)$ \\
\hline & Poor & $11(29.7)$ \\
\hline \multirow[t]{3}{*}{ T stage (AJCC, 7th ed.) } & $\mathrm{T} 1$ & $20(54.1)$ \\
\hline & $\mathrm{T} 2$ & $13(35.1)$ \\
\hline & T3 & $4(10.8)$ \\
\hline \multicolumn{2}{|c|}{ Tumor infiltration/compression of the major vasculature } & $9(24.3)$ \\
\hline \multicolumn{2}{|c|}{ Microvascular invasion of the tumor } & 9 (24.3) \\
\hline \multicolumn{2}{|l|}{ Presence of microsatellites } & 0 \\
\hline \multicolumn{2}{|l|}{ Liver capsule invasion of the tumor } & $22(59.5)$ \\
\hline \multicolumn{2}{|l|}{ Intraoperative blood transfusion } & $12(32.4)$ \\
\hline \multicolumn{2}{|l|}{ Postoperative bile leakage } & 0 \\
\hline \multicolumn{2}{|l|}{ 30-day operative mortality } & 0 \\
\hline The depth of IOERT (cm) & Median (min-max) & $0.9(0.8 \sim 1.2)$ \\
\hline \multirow[t]{3}{*}{ Applicator diameter for IOERT (cm) } & Median (min-max) & $5.5(3 \sim 10)$ \\
\hline & $\leq 5 \mathrm{~cm}$ & $19(51.4)$ \\
\hline & $>5 \mathrm{~cm}$ & $18(48.6)$ \\
\hline \multirow[t]{2}{*}{ IOERT energy } & $6 \mathrm{MV}$ & $28(75.7)$ \\
\hline & $9 \mathrm{MV}$ & $9(24.3)$ \\
\hline \multirow[t]{3}{*}{ Cone applicator angle for IOERT } & $0^{\circ}$ & $2(5.4)$ \\
\hline & $15^{\circ}$ & $7(18.9)$ \\
\hline & $30^{\circ}$ & $28(75.7)$ \\
\hline \multirow[t]{3}{*}{ IOERT dose } & 12-14 Gy & $3(8.1)$ \\
\hline & 15 Gy & 23 (62.2) \\
\hline & 16-17 Gy & $11(29.7)$ \\
\hline \multirow[t]{2}{*}{ Use of bolus } & $1 \mathrm{~cm}$ & $12(32.4)$ \\
\hline & $0.5 \mathrm{~cm}$ & 25 (67.6) \\
\hline
\end{tabular}

IOERT, Intraoperative Electron Radiotherapy; AFP, alpha-fetoprotein; AJCC, American Joint Committee on Cancer; WHO, World Health Organization; HBV, Hepatitis B virus.

grading $(P=0.076)$, staging $(P=0.818)$, tumor infiltration/ compression of the vasculature $(\mathrm{P}=0.931)$, tumor microvascular invasion $(\mathrm{P}=0.116)$, and tumor liver capsule invasion $(\mathrm{P}=0.47)$. Among the treatment parameters, the diameter of the IOERT applicator tube $(\mathrm{P}=0.057)$ and the IOERT energy $(\mathrm{P}=0.255)$ were not statistically significant risk factors with survival outcomes.

\section{Patterns of Recurrence}

During the follow-up, fifteen (40.5\%) patients suffered from a recurrence event (see Table 3). No patient had marginal recurrence. Twelve patients had intrahepatic recurrence, including nine patients with a single relapse, and three patients with multiple relapse. Three patients had extrahepatic recurrence, including one patient with multiple systemic metastases, one patient had intrahepatic relapse and peritoneal metastasis, and one patient had multiple bone metastases. There were nine patients $(24.3 \%)$ with early recurrence and six patients (16.2\%) with late recurrence. Eleven (29.7\%) patients received transarterial chemoembolization (TACE) therapy after recurrence. The 1-year, 3-year and 5-year intrahepatic recurrence rates were 19.75\%, 25.92\% and 39.58\%, respectively (see Figure 2C). The 1 -year, 3-year and 5-year extrahepatic recurrence rates were $2.7 \%$, $5.95 \%$ and $9.87 \%$, respectively (see Figure 2D).

\section{Toxicity}

Renal electrolyte, coagulation, and liver function were collected and analyzed preoperatively and postoperatively on days 1, 3, 5, 8 , and 14 , and on months $1,4,6,12$ and 18 . There were three patients with grade 4 , and 28 patients with grade 3 alanine aminotransferase (ALT) levels, and seven patients with grade 4, and 30 patients with grade a 3 aspartate transaminase (AST) levels. All of them returned to normal within four months (Figure 3). Gamma-glutamyl transferase (GGT) and alkaline phosphatase (ALP/AKP) values were grade 1-2 and none for grades 3-4. All the other indicators were grade 1-2, except for one grade 3 patient with an activated partial coagulation time (APTT) who returned to normal within two weeks (Figure 3). None of the patients who received IOERT developed RILD. There were no acute or chronic toxicities associated with IOERT.

\section{DISCUSSION}

To the best of our knowledge, this is the first study to illustrate the long-term survival and safety of adjuvant therapy with IOERT for CL-HCC. Our data revealed that IOERT is a potentially viable adjuvant therapy for CL-HCC patients with a narrow-margin resection.

\section{Feasibility Considerations}

Most studies have confirmed that insufficient margins can have a $16-50 \%$ recurrence rate (23-29). However, because the tumor is adjacent to major vessels for CL-HCC patients, narrow-margin resection is the only option, currently. CL-HCC with a $\mathrm{RM}<1 \mathrm{~cm}$ has an unfavorable prognosis. Several studies have found that residual microscopic disease commonly occurs around the primary tumour (30-33). The main reason for early recurrence are residual microscopic lesions, a subclinical focus in the postoperative tumor bed, and peri-hepatic tumor minification metastasis by partial microvascular invasion (2, 3). Early recurrence is usually multi-focal or multi-nodule recurrence, so it is difficult to perform radical salvageable treatment (34). Therefore, the goal of reducing marginal and early recurrence may be achieved through microscopic lesion control. Effective adjuvant therapy targeting the narrow margin is the key to eradicating microscopic lesions around the main tumor.

Hepatocellular carcinoma cells have a high sensitivity to radiation, which could mean a similar control rate to squamous cell carcinoma is possible. The dosimetry advantages and clinical benefits of IOERT have been demonstrated among 
A

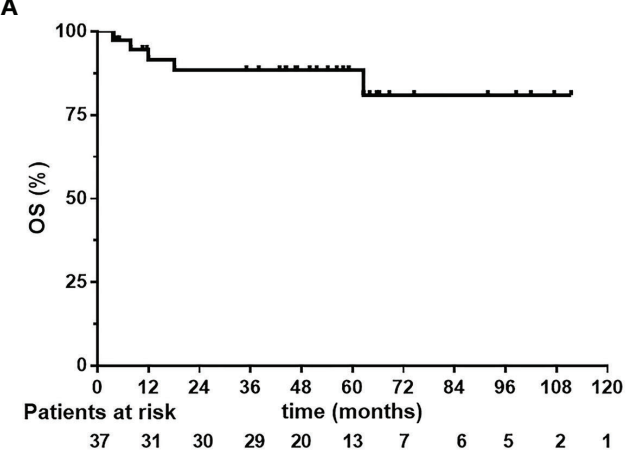

C

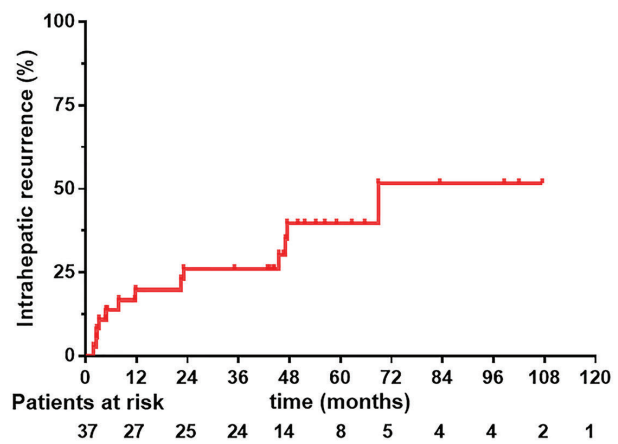

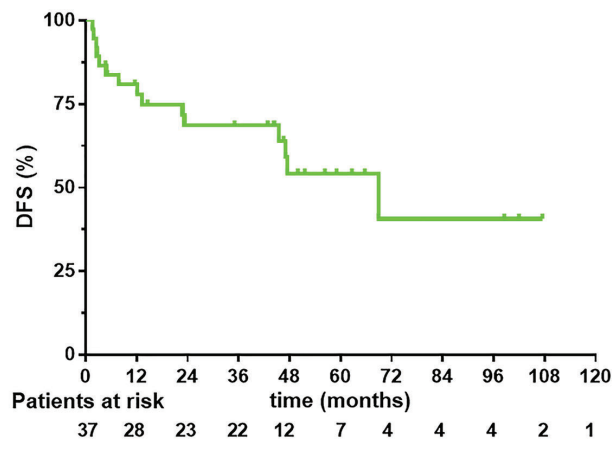

D

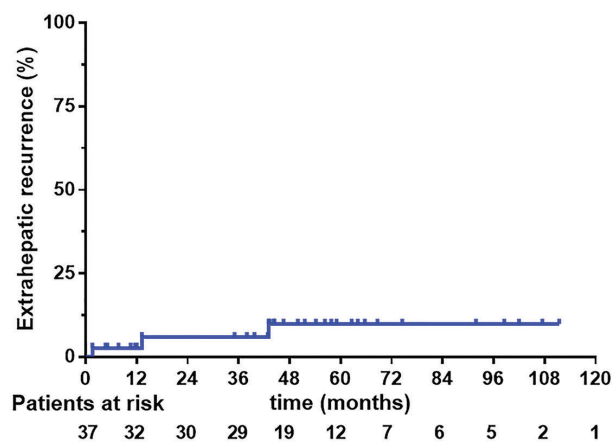

FIGURE 2 | Overall survival (OS), disease-free survival (DFS) (A, B) and intrahepatic and extrahepatic recurrence curves (C, D) 37 CL-HCC patients received narrow-margin resection combined with IOERT. IOERT, Intraoperative Electron Radiotherapy.

TABLE 2 | Univariate analysis for overall survival.

\begin{tabular}{lcc}
\hline Prognostic factors & HR & $\mathbf{9 5 \%} \mathbf{C l}$ \\
\hline Age (years) (>60 vs. $\leq 60)$ & 0.304 & $0.033-2.773$ \\
Gender (male vs. female) & 0.312 & $0.032-3.086$ \\
HBV (positive vs. negative) & 37.597 & 0.291 \\
Long-term drinker (yes vs. no) & 1.559 & $0.013-107956.335$ \\
AFP value (ug/L) (>400 vs. $\leq 400)$ & 306.309 & $0.172-14.105$ \\
Tumor size (cm) (>5 vs. $\leq 5)$ & 3.581 & $0.023-4047801.226$ \\
Histological grading (WHO) (well-moderate vs. poor) & 0.195 & $0.578-22.190$ \\
Stage (AJCC, 7th ed.) (T2-3 vs. T1) & 1.240 & $0.320-1.185$ \\
Tumor infiltration/compression of the vasculature (yes vs. no) & 1.102 & $0.198-7.761$ \\
Microvascular invasion (yes vs. no) & 4.379 & $0.120-10.088$ \\
Liver capsule invasion (yes vs. no) & 0.444 & $0.695-27.589$ \\
Diameter cone applicator of IOERT (cm) (>5 vs. $\leq 5)$ & 10.235 & $0.049-4.018$ \\
IOERT energy (16 17Gy vs. 15Gy) & 3.287 & $0.937-111.802$ \\
\hline
\end{tabular}

IOERT, Intraoperative Electron Radiotherapy; AFP, alpha-fetoprotein; AJCC, American Joint Committee on Cancer; WHO, World Health Organization; HBV, Hepatitis B virus; Cl, confidence interval; HR, hazard ratio.

various malignancies (10-18). In our study there were 15 (40.5\%) patients with recurrence, nine of which $(24.3 \%)$ experienced early recurrence, but no patients had marginal recurrence compared with $\mathrm{Yu} \mathrm{W}$, et al., who reported a recurrence rate of $50.8 \%$ after resection alone (35). Similar results were also reported by Wang $\mathrm{WH}$, et al., who showed that a narrowmargin resection for CL-HCC with a margin recurrence of $9.6 \%$ and an early recurrence of $43.4 \%$ (36). Although the results were compared in a single center, the treatment techniques and indications were similar and comparable. These studies indicate that narrow-margin resection combined with IOERT may reduce marginal and early recurrence.

Our study also shows favorable outcomes. The gain in survival is partially attributed to the reduction in marginal and early recurrence. In our study, the 1-year, 3-year and 5-year OS rates were $91.39 \%, 88.34 \%$ and $88.34 \%$, respectively. The 1 -year, 3 -year and 5-year DFS rates were $80.81 \%, 68.59 \%$ and $54.17 \%$, respectively. These results compare favorably to the results of 
TABLE 3 | Pattern of recurrence.

\begin{tabular}{lc} 
Variable & No. of patients (\%) \\
\hline Total recurrence & $15(40.5)$ \\
Time to recurrence & \\
$\quad$ early recurrence ( $\leq 18$ months) & $9(24.3)$ \\
late recurrence (>18 months) & $6(16.2)$ \\
Intrahepatic recurrence & $12(32.4)$ \\
marginal recurrence & 0 \\
single nodule & $9(24.3)$ \\
Multiple nodules & $3(8.1)$ \\
Extrahepatic recurrence & $3(8.1)$ \\
Treatment after recurrence & \\
TACE & $11(29.7)$ \\
RFA & $1(2.7)$ \\
Chemotherapy & $1(2.7)$ \\
Molecular targeted therapy & $1(2.7)$ \\
surgery & $1(2.7)$ \\
\hline
\end{tabular}

TACE, trans-arterial chemoembolization; RFA, radiofrequency ablation.

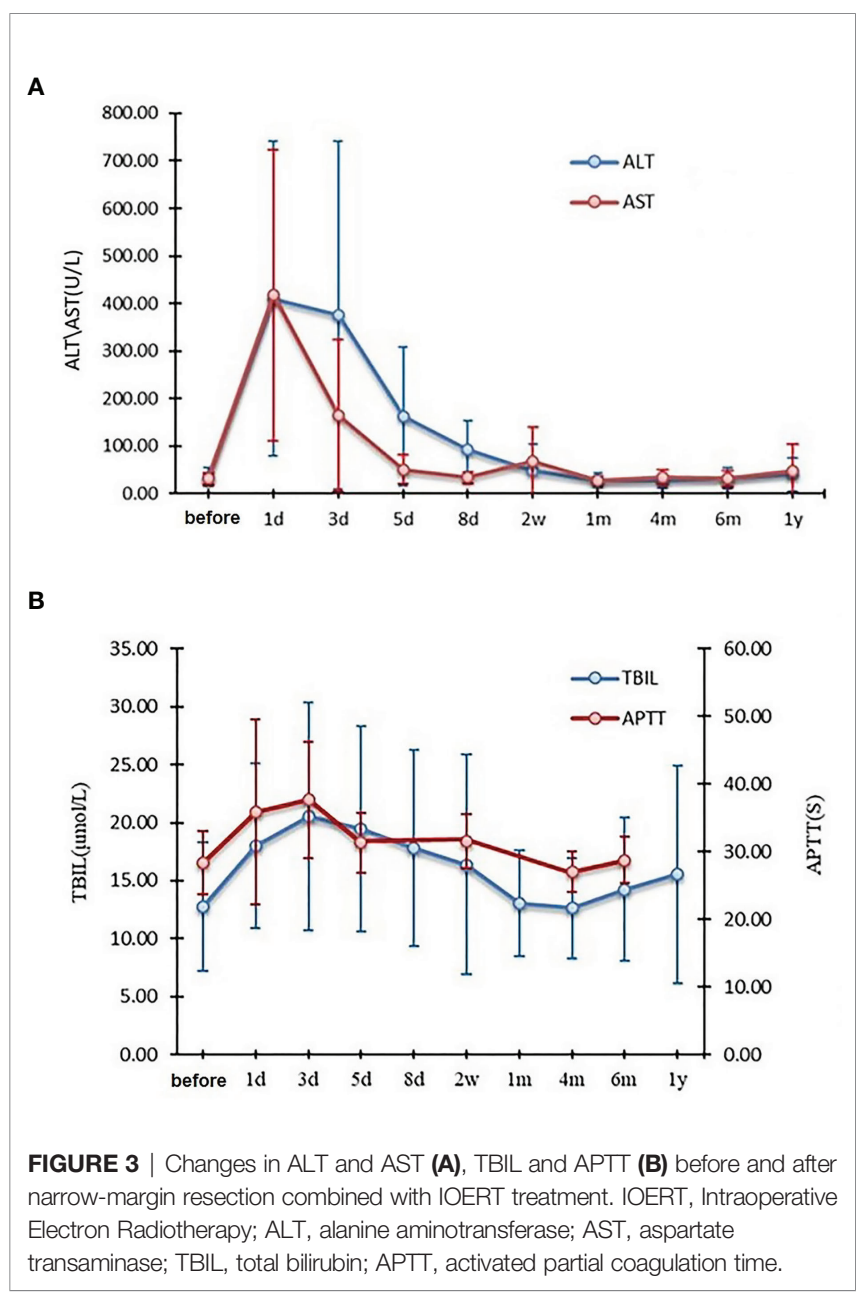

$\mathrm{Yu}$ W, et al. during the same period and in the same hospital, with $74.5 \%$ and $40.1 \%$ for the 3 -year OS and DFS using surgery alone, respectively (35). The 3 -year OS and DSF increased by $13.84 \%$ and $28.49 \%$, respectively. Wang $\mathrm{WH}$, et al. retrospectively analyzed the results of $181 \mathrm{CL}-\mathrm{HCC}$ with IMRT in the same hospital. The results showed that the 3-year OS with a narrow-margin in the IMRT group, and surgery only group, and the wide-margin $(>1 \mathrm{~cm})$ group, were $89.1 \%, 67.7 \%$ and $86.0 \%$ ( $\mathrm{P}=0.009$ ), respectively, and the 3 -year DFS was $64.2 \%$, $52.2 \%$ and $60.1 \%(\mathrm{P}=0.038)$, respectively. Postoperative radiotherapy may improve the effect in patients with narrow incisions (36). Based on these trials and our data, IOERT appears to control the residual tumor at the resection margin and persistent residual microscopic lesions in the remnant liver tissue surrounding primary HCC, which should decrease the risk of early intrahepatic and extrahepatic spread through the portal and hepatic veins. For patients at our institute with narrow margin hepatectomy, we recommend IOERT.

\section{Safety Considerations}

While IOERT provides an opportunity for intraoperative adjuvant radiotherapy, it is difficult to preserve normal liver function. In our 37-patient study, most (95\%) had multiple risk factors: fourteen patients had a tumor diameter $>5 \mathrm{~cm}$, nine patients had tumor infiltration/compression of the major vasculature, nine patients had microvascular invasion of the tumor, and 34 patients had cirrhosis. Only one patient developed acute renal failure on the second day after surgery and returned to normal after one week in intensive care. Although there were changes in liver function before and after surgery, AST and ALT returned to normal within four months. There was no RILD found during follow-up.

Two small studies have reported on postoperative complications after IOERT adjuvant therapy. In a similar case control analysis by $\mathrm{Xu} \mathrm{C}$, et al. the liver function indicators in the two groups increased and recovered in a similar manner ( $\mathrm{P}>0.05)$, without RILD occurence (37). Liu YH, et al. used a prospective cohort study to analyze the results of CL-HCC combined with IOERT, which was similar to our findings. With a prolonged surgical time, IOERT was safe and feasible (38). Taking all these findings into account, we conclude that IOERT adjuvant therapy does not increase the postoperative complication rate as was commonly thought. IOERT is safe as an adjuvant treatment for CL-HCC patients.

In the univariate analysis, none of the patients, tumor characteristics or IOERT parameters were predictive of overall survival. Since 2009, we have employed IOERT energy between 12 and $17 \mathrm{~Gy}$ combined with adjuvant treatment without causing serious side effects. The current target volume and dose of IOERT may be suitable to improve tumor control despite the limited number of patients analyzed. We suggest that IOERT at $15 \mathrm{~Gy}$, at present, is the most appropriate dose as more energy does not appear to convey anymore benefits.

\section{Limitations}

This was a retrospective cohort study using prospectively collected data, which minimized potential differential information bias. However, there are still some limitations. This was a single-institutional analysis. Therefore, the associated limitations need to be considered when interpreting these results. Besides, this pilot study included only a small number of patients receiving IOERT following narrow-margin 
resection for CL-HCC. It is difficult to adequately adjust for all potential confounding factors. However, the data does provide a rationale for developing well-designed clinical trials.

\section{CONCLUSIONS}

This study revealed that IOERT as an adjuvant therapy for narrow-margin CL-HCC may achieve good long-term survival outcomes, without significantly increasing toxic side effects. It is suggested that an IOERT dose of 15Gy may be administered immediately after the tumor is resected with a surgical margin of approximately $1 \mathrm{~cm}$. However, prospective studies with larger patient cohorts are needed to confirm these results.

\section{DATA AVAILABILITY STATEMENT}

The original contributions presented in the study are included in the article/supplementary material. Further inquiries can be directed to the corresponding author.

\section{ETHICS STATEMENT}

The studies involving human participants were reviewed and approved by the ethics committee of the Cancer Hospital of the

\section{REFERENCES}

1. Siegel RL, Fedewa SA, Miller KD, Goding-Sauer A, Pinheiro PS, MartinezTyson D, et al. Cancer Statistics for Hispanics/Latinos, 2015. CA Cancer J Clin (2015) 65(6):457-80. doi: 10.3322/caac.21314

2. Bruix J, Sherman M. Management of Hepatocellular Carcinoma: An Update. Hepatology (2011) 53:1020-2. doi: 10.1002/hep.24199

3. Kobayashi T, Ishiyama K, Ohdan H. Prevention of Recurrence After Curative Treatment for Hepatocellular Carcinoma. Surg Today (2013) 43(12):1347-54. doi: 10.1007/s00595-012-0473-5

4. Matsui Y, Terakawa N, Satoi S, Kaibori M, Kitade H, Takai S, et al. Postoperative Outcomes in Patients With Hepatocellular Carcinomas Resected With Exposure of the Tumor Surface, Clinical Role of the Nomargin Resection. Arch Surg (2007) 142:596-602. doi: 10.1001/archsurg. 142.7.596

5. Cheng CH, Yu MC, Wu TH, Lee CF, Chan KM, Chou HS, et al. Surgical Resection of Centrally Located Large Hepatocellular Carcinoma. Chang Gung Med J (2012) 35:178-91. doi: 10.4103/2319-4170.106153

6. Rim CH, Seong J. Application of Radiotherapy for Hepatocellular Carcinoma in Current Clinical Practice Guidelines. Radiat Oncol J (2016) 34(3):160-7. doi: 10.3857/roj.2016.01970

7. Hawkins MA, Dawson LA. Radiation Therapy for Hepatocellular Carcinoma: From Palliation to Cure. Cancer (2006) 106:1653-63. doi: 10.1002/cncr.21811

8. Krishnan S, Dawson LA, Seong J, Akine Y, Beddar S, Briere TM, et al. Radiotherapy for Hepatocellular Carcinoma: An Overview. Ann Surg Oncol (2008) 15:1015-24. doi: 10.1245/s10434-007-9729-5

9. Jihye C, Jinsil S. Application of Radiotherapeutic Strategies in the BCLCdefined Stages of Hepatocellular Carcinoma. Liver Cancer (2012) 1:216-25. doi: $10.1159 / 000343836$

10. König L, Lang K, Heil J, Golatta M, Major G, Krug D, et al. Acute Toxicity and Early Oncological Outcomes After Intraoperative Electron Radiotherapy (IOERT) as Boost Followed by Whole Breast Irradiation in 157 Early Stage
Chinese Academy of Medical Sciences. The patients/participants provided their written informed consent to participate in this study.

\section{AUTHOR CONTRIBUTIONS}

Contributorship statement study concepts: Y-lW, Y-xL, J-xW, Q-fF, and J-qC. Study design: Y-rZ, M-hL, J-qC, PM, L-mW, and X-hW. Data acquisition: Y-lW, Q-fF, FW. Quality control of data and algorithms: M-hL, J-qC, PM, L-mW, X-hW, J-xW, and Q-fF. Data analysis and interpretation: Y-lW, Y-rZ, J-xW, and Q-fF. Statistical analysis: M-hL, PM, L-mW, X-dW, FW, QZ, and BC. Manuscript preparation: Y-lW, M-hL, J-qC, and PM. Manuscript editing: Y-lW and Y-rZ. Manuscript review: Y-xL, J-qC, J-xW, and Q-fF. All authors contributed to the article and approved the submitted version.

\section{FUNDING}

The study was supported by the Clinical Application Project of Beijing Municipal Science \& Technology Commission (Z1107058811093), and the Beijing Municipal Science and Technology Project (Z131107002213166). The funders had no role in study design, data collection and analysis, decision to publish, or preparation of the manuscript.

Breast Cancer Patients-First Clinical Results From a Single Center. Front Oncol (2019) 9:384. doi: 10.3389/fonc.2019.00384

11. Kaiser J, Reitsamer R, Kopp P, Gaisberger C, Kopp M, Fischer T, et al. Intraoperative Electron Radiotherapy (IOERT) in the Treatment of Primary Breast Cancer. Breast Care (Basel) (2018) 13(3):162-7. doi: 10.1159/000489637

12. Chen Y, Che X, Zhang J, Huang H, Zhao D, Tian Y, et al. Long-Term Results of Intraoperative Electron Beam Radiation Therapy for Nonmetastatic Locally Advanced Pancreatic Cancer. Med (Baltimore) (2016) 95(38):e4861. doi: 10.1097/MD.0000000000004861

13. Voogt ELK, van Rees JM, Hagemans JAW, Rothbarth J, Nieuwenhuijzen GAP, Cnossen JS, et al. Intraoperative Electron Beam Radiation Therapy (IOERT) Versus High-Dose-Rate Intraoperative Brachytherapy (HDR-IORT) in Patients With an R1 Resection for Locally Advanced or Locally Recurrent Rectal Cancer. Int J Radiat Oncol Biol Phys (2021) 110(4):1032-43. doi: 10.1016/j.ijrobp.2021.02.006

14. Kyrgias G, Hajiioannou J, Tolia M, Kouloulias V, Lachanas V, Skoulakis C, et al. Intraoperative Radiation Therapy (IORT) in Head and Neck Cancer: A Systematic Review. Medicine (2016) 95(50):e5035. doi: 10.1097/MD. 0000000000005035

15. Soriani A, Landoni V, Marzi S, Iaccarino G, Saracino B, Arcangeli G, et al. Setup Verification and In Vivo Dosimetry During Intraoperative Radiation Therapy (IORT) for Prostate Cancer. Med Phys (2007) 34:3205-10. doi: 10.1118/1.2750965

16. Fu S, Lu JJ, Zhang Q, Yang Z, Peng L, Xiong F. Intraoperative Radiotherapy Combined With Adjuvant Chemoradiotherapy for Locally Advanced Gastric Adenocarcinoma. Int J Radiat Oncol Biol Phys (2008) 72:1488-94. doi: 10.1016/j.ijrobp.2008.03.012

17. Roeder F, Alldinger I, Uhl M, Saleh-Ebrahimi L, Schimmack S, Mechtersheimer G, et al. Intraoperative Electron Radiation Therapy in Retroperitoneal Sarcoma. Int J Radiat Oncol Biol Phys (2018) 100:516-27. doi: 10.1016/j.ijrobp.2017.10.034

18. Roeder F, de Paoli A, Saleh-Ebrahimi L, Alldinger I, Bertola G, Boz G, et al. Intraoperative Electron Radiation Therapy Combined With External Beam 
Radiation Therapy After Gross Total Resection in Extremity Soft Tissue Sarcoma: A European Pooled Analysis. Ann Surg Oncol (2018) 25:3833-42. doi: 10.1245/s10434-018-6787-9

19. Benson AB 3rd, Abrams TA, Ben-Josef E, Bloomston PM, Botha JF, Clary BM, et al. NCCN Clinical Practice Guidelines in Oncology: Hepatobiliary Cancers. J Natl Compr Canc Netw (2009) 7(4):350-91. doi: 10.6004/jnccn.2009.0027

20. Lawrence TS, Ten Haken RK, Kessler ML, Robertson JM, Lyman JT, Lavigne ML, et al. The Use of 3-D Dose Volume Analysis to Predict Radiation Hepatitis. Int J Radiat Oncol Biol Phys (1992) 23(4):781-8. doi: 10.1016/0360-3016(92)90651-W

21. Trotti A, Byhardt R, Stetz J, Gwede C, Corn B, Fu K, et al. Common Toxicity Criteria: Version 2.0. An Improved Reference for Grading the Acute Effects of Cancer Treatment: Impact on Radiotherapy. Int J Radiat Oncol Biol Phys (2000) 47(1):13-47. doi: 10.1016/S0360-3016(99)00559-3

22. Huang ZY, Liang BY, Xiong M, Zhan DQ, Wei S, Wang GP, et al. Long-Term Outcomes of Repeat Hepatic Resection in Patients With Recurrent Hepatocellular Carcinoma and Analysis of Recurrent Types and Their Prognosis: A Single-Center Experience in China. Ann Surg Oncol (2012) 19:2515-25. doi: 10.1245/s10434-012-2269-7

23. Jeng KS, Jeng WJ, Sheen IS, Lin CC, Lin CK. Is Less Than $5 \mathrm{Mm}$ as the Narrowest Surgical Margin Width in Central Resections of Hepatocellular Carcinoma Justifified? Am J Surg (2013) 206:64-71. doi: 10.1016/ j.amjsurg.2012.06.010

24. Chau GY, Lui WY, Tsay SH, King KL, Loong CC, Chiu JH, et al. Prognostic Signifificance of Surgical Margin in Hepatocellular Carcinoma Resection: An Analysis of 165 Childs' A Patients. J Surg Oncol (1997) 66:122-6. doi: 10.1002/ (SICI)1096-9098(199710)66:2<122::AID-JSO9>3.0.CO;2-F

25. Hu RH, Lee PH, Chang YC, Ho MC, Yu SC. Treatment of Centrally Located Hepatocellular Carcinoma With Central Hepatectomy. Surgery (2003) 133:251-6. doi: 10.1067/msy.2003.102

26. Ikai I, Arii S, Kojiro M, Ichida T, Makuuchi M, Matsuyama Y, et al. Reevaluation of Prognostic Factors for Survival After Liver Resection in Patients With Hepatocellular Carcinoma in a Japanese Nationwide Survey. Cancer (2004) 101:796-802. doi: 10.1002/cncr.20426

27. Shi M, Guo RP, Lin XJ, Zhang YQ, Chen MS, Zhang CQ, et al. Partial Hepatectomy With Wide Versus Narrow Resection Margin for Solitary Hepatocellular Carcinoma: A Prospective Randomized Trial. Ann Surg (2007) 245:36-43. doi: 10.1097/01.sla.0000231758.07868.71

28. Nanashima A, Sumida Y, Abo T, Nagasaki T, Tobinaga S, Fukuoka H, et al. Comparison of Survival Between Anatomic and non-Anatomic Liver Resection in Patients With Hepatocellular Carcinoma: Signifificance of Surgical Margin in non-Anatomic Resection. Acta Chir Belg (2008) 108:532-7. doi: 10.1080/00015458.2008.11680280

29. Hirokawa E, Hayashi M, Miyamoto Y, Asakuma M, Shimizu T, Komeda K, et al. Outcomes and Predictors of Microvascular Invasion of Solitary Hepatocellular Carcinoma. Hepatol Res (2014) 44:846-53. doi: 10.1111/ hepr.12196

30. Wang WH, Feng X, Zhang T, Jin J, Wang S, Liu Y, et al. Prospective Evaluation of Microscopic Extension Using Whole-Mount Preparation in Patients With Hepatocellular Carcinoma: Definition of Clinical Target
Volume for Radiotherapy. Radiat Oncol (2010) 5:73. doi: 10.1186/1748717X-5-73

31. Shi M, Zhang CQ, Zhang YQ, Liang XM, Li JQ. Micrometastasis of Solitary Hepatocellular Carcinoma and Appropriate Resection Margin. World J Surg (2004) 28:376-81. doi: 10.1007/s00268-003-7308-x

32. Zhou XP, Quan ZW, Cong WM, Yang N, Zhang HB, Zhang SH, et al. Micrometastasis in Surrounding Liver and the Minimal Length of Resection Margin of Primary Liver Cancer. World J Gastroenterol (2007) 13:4498-503. doi: 10.3748/wjg.v13.i33.4498

33. Okusaka T, Okada S, Ueno H, Ikeda M, Shimada K, Yamamoto J, et al. Satellite Lesions in Patients With Small Hepatocellular Carcinoma With Reference to Clinicopathologic Features. Cancer (2002) 95:1931-7. doi: 10.1002/cncr.10892

34. Zhang XF, Beal EW, Bagante F, Chakedis J, Weiss M, Popescu I, et al. Early Versus Late Recurrence of Intrahepatic Cholangiocarcinoma After Resection With Curative Intent. Br J Surg (2017) 105:848-56. doi: 10.1002/bjs.10676

35. Yu W, Wang W, Rong W, Wang L, Xu Q, Wu F, et al. Adjuvant Radiotherapy in Centrally Located Hepatocellular Carcinomas After Hepatectomy With Narrow Margin (<1 Cm): A Prospective Randomized Study. J Am Coll Surg (2014) 218(3):381-92. doi: 10.1016/j.jamcollsurg.2013.11.030

36. Wang WH, Wang Z, Wu JX, Zhang T, Rong WQ, Wang LM, et al. Survival Benefit With IMRT Following Narrow-Margin Hepatectomy in Patients With Hepatocellular Carcinoma Close to Major Vessels. Liver Int (2015) 35 (12):2603-10. doi: 10.1111/liv.12857

37. Xu C, feng QF, Bi XY, Fan CC, Zhai YR, Li MH, et al. Safety of Intraoperative Radiotherapy for HCC. Chin J Radiat Oncol (2014) 23(5):386-90. doi: 10.3760/cma.j.issn.1004-4221.2014.05.004

38. Liu YH, Wang LL, Wu JH, Rong WQ, Wu F, Li MH, et al. A Prospective and Preliminary Study of Central Hepatocellular Carcinoma Combined With Intraoperative Tumor Bed Radiotherapy. Chin J Oncol (2017) 39(12):92630. doi: 10.3760/cma.j.issn.0253-3766.2017.12.009

Conflict of Interest: The authors declare that the research was conducted in the absence of any commercial or financial relationships that could be construed as a potential conflict of interest.

Publisher's Note: All claims expressed in this article are solely those of the authors and do not necessarily represent those of their affiliated organizations, or those of the publisher, the editors and the reviewers. Any product that may be evaluated in this article, or claim that may be made by its manufacturer, is not guaranteed or endorsed by the publisher.

Copyright (C) $2022 \mathrm{Wu}$, Zhai, Li, Cai, Ma, Wang, Wu, Wang, Wu, Zeng, Chen, Li, Wu and Feng. This is an open-access article distributed under the terms of the Creative Commons Attribution License (CC BY). The use, distribution or reproduction in other forums is permitted, provided the original author(s) and the copyright owner(s) are credited and that the original publication in this journal is cited, in accordance with accepted academic practice. No use, distribution or reproduction is permitted which does not comply with these terms. 